\title{
Le développement de compétences professionnelles par des enseignants en exercice : le cas de l'évaluation des apprentissages
}

\section{The development of professional competencies by practicing teachers: learning assessment El desarrollo de competencias profesionales por maestros en ejercicio: el caso de la evaluación de aprendizajes}

\section{Isabelle Nizet}

Volume 44, numéro 2, automne 2016

Le développement de compétences en éducation et en formation

URI : https://id.erudit.org/iderudit/1039024ar

DOI : https://doi.org/10.7202/1039024ar

Aller au sommaire du numéro

\section{Éditeur(s)}

Association canadienne d'éducation de langue française

ISSN

1916-8659 (numérique)

Découvrir la revue

Citer cet article

Nizet, I. (2016). Le développement de compétences professionnelles par des enseignants en exercice : le cas de l'évaluation des apprentissages. Éducation et francophonie, 44(2), 109-125. https://doi.org/10.7202/1039024ar

\section{Résumé de l'article}

Dans le contexte de la mise en oeuvre de programmes par compétences en enseignement secondaire au Québec, nous analysons le processus de construction de savoirs professionnels d'enseignants du secteur de l'éducation des adultes, dans le cadre d'une recherche-formation collaborative qui a duré deux ans et qui était centrée exclusivement sur le développement de la compétence professionnelle à évaluer les apprentissages. Les enseignants y étaient invités à se former pour former leurs pairs. À partir de l'analyse de contenu d'extraits d'échanges ayant eu lieu durant quatre journées de formation sur un ensemble de vingt-sept, nous avons réalisé, une fois le projet terminé, une étude visant à repérer les mécanismes par lesquels les participants attribuent une valeur aux savoirs de formation. L'étude a révélé la difficulté des enseignants à construire des savoirs professionnels de référence, alors que les savoirs issus de leur expérience jouissent a priori d'une crédibilité et d'une légitimité plus grandes que les savoirs construits dans un espace de formation. Ce constat nous amène à interroger les conditions de développement de compétences professionnelles par des enseignants en exercice.
Tous droits réservés (C) Association canadienne d'éducation de langue française, 2017 services d'Érudit (y compris la reproduction) est assujettie à sa politique d'utilisation que vous pouvez consulter en ligne. 


\section{Le développement de compétences professionnelles par des enseignants en exercice: le cas de l'évaluation des apprentissages}

\section{Isabelle NIZET}

Université de Sherbrooke, Québec, Canada

\section{RÉSUMÉ}

Dans le contexte de la mise en œuvre de programmes par compétences en enseignement secondaire au Québec, nous analysons le processus de construction de savoirs professionnels d'enseignants du secteur de l'éducation des adultes, dans le cadre d'une recherche-formation collaborative qui a duré deux ans et qui était centrée exclusivement sur le développement de la compétence professionnelle à évaluer les apprentissages. Les enseignants y étaient invités à se former pour former leurs pairs. À partir de l'analyse de contenu d'extraits d'échanges ayant eu lieu durant quatre journées de formation sur un ensemble de vingt-sept, nous avons réalisé, une fois le projet terminé, une étude visant à repérer les mécanismes par lesquels les participants attribuent une valeur aux savoirs de formation. L'étude a révélé la difficulté des enseignants à construire des savoirs professionnels de référence, alors que les savoirs issus de leur expérience jouissent $a$ priori d'une crédibilité et d'une légitimité plus grandes que les savoirs construits dans un espace de formation. Ce constat nous 
Le développement de compétences professionnelles par des enseignants en exercice:

le cas de l'évaluation des apprentissages

amène à interroger les conditions de développement de compétences professionnelles par des enseignants en exercice.

\section{ABSTRACT}

\section{The development of professional competencies by practicing teachers: learning assessment}

Isabelle NIZET, University of Sherbrooke, Québec, Canada

In the context of the implementation of secondary-level competency programs in Québec, we analyze the process of building professional knowledge among adulteducation teachers as part of a collaborative research-training that lasted 2 years and was exclusively focused on developing the professional competency of learning assessment. The teachers were invited to take training to teach their peers. Once the project was finished, through the analysis of content drawn from exchanges that took place during four out of a total of twenty-seven days of training, we conducted a study to identify mechanisms through which participants attributed a value to the training knowledge. The study revealed the trouble teachers had building professional reference knowledge, while the knowledge that came from their experience had greater credibility and legitimacy than the knowledge built in a training space. This observation led us to question conditions practicing teachers require for the development of professional competencies.

\section{RESUMEN}

\section{El desarrollo de competencias profesionales por maestros en ejercicio: el caso de la evaluación de aprendizajes}

Isabelle NIZET, Universidad de Sherbrooke, Quebec, Canadá

En el contexto de la aplicación de programas por competencias en enseñanza secundaria en Quebec, analizamos el proceso de construcción de saberes profesionales de maestros del sector de la educación de los adultos, en el marco de una investigaciónformación colaborativa que duró dos años y que estaba centrada exclusivamente en el desarrollo de la competencia profesional para evaluar los aprendizajes. Los maestros fueron invitados a formarse con el fin de formar a sus pares. A partir de los análisis de contenido extraídos de los intercambios que hubo durante los cuatro días de formación sobre un conjunto de veintisiete, hemos realizado, una vez el proyecto terminado, un estudio cuyo objetivo era identificar los mecanismos utilizados por los participantes para atribuir un valor a los conocimientos para formar. El estudio mostró la dificultad de los maestros para construir los saberes profesionales de referen- 
cia, mientras que los saberes provenientes de su experiencia gozaban a priori de una credibilidad y de una legitimidad mayor que los saberes construidos durante la formación. Esta constatación nos lleva a interrogarnos sobre las condiciones de desarrollo de competencias profesionales entre los maestros en ejercicio.

\section{INTRODUCTION}

Au Québec, la formation continue des enseignants en exercice est considérée comme une obligation individuelle et collective. En réponse à des besoins de formation identifiés au niveau local, l'offre de formation continue est gérée par les commissions scolaires; ses visées essentielles sont le «maintien des compétences», la mise à jour et l'enrichissement de la pratique professionnelle par des moyens variés dans lesquels l'expertise et l'expérience des enseignants sont reconnues (Conseil supérieur de l'éducation, 2014; Gouvernement du Québec, 1999). En tant que partenaires intervenant dans la formation continue formelle des enseignants, les équipes universitaires joignent de plus en plus fréquemment à ces dispositifs de formation un processus de recherche de type collaboratif et la mise en place de groupes de travail structurés en communautés d'apprentissage (Fontaine, Savoie-Zajc et Cadieux, 2013; Vinatier, 2015). Dans cette perspective d'articulation entre formation et recherche, nous avons mis en place de 2013 à 2015 et sur deux sites distincts un dispositif de formation continue à caractère collaboratif intitulé Soutien au développement de pratiques évaluatives d'enseignants de la formation générale des adultes pour 14 enseignantes et enseignants et 5 conseillères et conseillers pédagogiques de la formation générale des adultes (FGA) dans quatre commissions scolaires du Québec. Ce projet a été financé dans le cadre du Programme de soutien à la formation continue du personnel enseignant du ministère de l'Éducation, du Loisir et du Sport, programme qui existe depuis 2009.

Les résultats des recherches collaboratives mettent de plus en plus l'accent sur la description et la compréhension des processus de construction de savoirs professionnels in situ et sur leur émergence dans une grande diversité de domaines de formation, qu'il s'agisse des enseignants ou d'autres professionnels des métiers de l'humain (Desgagné, Bednarz, Lebuis, Poirier et Couture, 2001; Ducharme, Leblanc, Bourassa et Chevalier, 2007; Morrissette, Mottier Lopez et Tessaro, 2012; Vinatier, 2015). Échelonnée sur un plus ou moins long laps de temps, la formation continue est donc considérée comme un terrain propice à l'observation du développement de compétences des enseignants qui réfléchissent sur leurs situations professionnelles et sur les apprentissages réalisés au sein de ces groupes. 
Au terme du projet de formation continue mentionné plus haut, nous avons éprouvé le besoin de réfléchir sur les conditions qui ont pu favoriser ou freiner la construction de savoirs professionnels par les enseignants en ce qui concerne leur compétence à évaluer les élèves. Afin de mener cette étude, nous avons élaboré un cadre conceptuel prenant appui sur des concepts de didactique professionnelle tels que «savoir professionnel», «savoir de référence» et "construction de savoirs professionnels» (Vanhulle, 2013, 2014). Nous avons également développé une réflexion de nature épistémologique portant sur les critères de validité et de viabilité attribuées aux savoirs de formation par les participants à des projets collaboratifs (Nizet et Leroux, 2015). Ce cadre a été mobilisé pour problématiser a posteriori les observations empiriques réalisées durant le projet de formation continue dont il est question ici et pour repérer les valeurs attribuées aux savoirs de formation en lien avec deux problèmes de formation abordés dans la recherche-formation collaborative. Les résultats de cette étude seront discutés afin de produire des pistes de réflexion sur les conditions favorables au développement de la compétence à évaluer en contexte collaboratif.

\section{CONTEXTE}

Le contexte de notre étude se situe à l'éducation des adultes, dont les enseignants sont depuis 2007 aux prises avec la mise en œuvre de programmes par compétences, ce qui crée chez eux d'importants besoins de formation continue. L'ancien curriculum fondé sur une approche par objectifs est remplacé depuis 2007 en formation de base commune $(\mathrm{FBC})^{1}$ et depuis 2011 en formation de base diversifiée, pour les élèves adultes $(\mathrm{FBD})^{2}$, par un curriculum structuré autour de compétences situationnelles et polyvalentes en FBC, disciplinaires et transversales en FBD. En résumé, l'introduction de ces nouveaux programmes modifie le rapport de l'enseignant aux savoirs enseignés dans des modules d'autoapprentissage, l'incitant à passer d'une expertise disciplinaire centrée sur les connaissances à une expertise centrée sur les compétences. Les changements curriculaires impliquent le renouvellement du matériel et la diversification des modalités pédagogiques, alors que les enseignants n'ont pas tous pu développer une expérience à cet égard. Le matériel d'apprentissage standardisé ne peut plus servir d'appui pour l'accompagnement des élèves qu'il faut préparer à la passation d'examens certificatifs dont le contenu est profondément modifié, notamment par l'introduction de situations d'évaluation dans lesquelles les élèves doivent démontrer leur compétence en traitant des tâches complexes. L'évaluation des connaissances y prend une place limitée au profit de l'évaluation des manifestations de compétences, objets difficiles à cerner pour des enseignants habitués à procéder à une correction objective de connaissances et non formés en

1. Couvrant les huit premières années de formation et comprenant l'alphabétisation, le présecondaire et le premier cycle du secondaire.

2. Couvrant les trois années du second cycle du secondaire, accessible aux élèves de 16 ans et plus (limite de la scolarité obligatoire au Québec) qui n'ont pas obtenu leur diplôme d'études secondaires. 
évaluation (Nizet, 2014). À l'ensemble de ces modifications s'ajoute la nécessité de développer des démarches d'évaluation «en aide à l'apprentissage» (Gouvernement du Québec, 2003), qui favorisent un apprentissage durable chez les élèves adultes.

Ces éléments caractérisent de manière générale les situations professionnelles des enseignants de FGA et délimitent la nature des problèmes de formation abordés au cours du projet. Nous avons choisi un modèle de formation de type non applicatif pour donner une place prépondérante aux acteurs et laisser une place importante à leur réalité professionnelle (Boudjaoui et Clénet, 2011). Ce choix est de plus en plus fréquent lorsqu'il s'agit de former les enseignants à l'évaluation (Durand et Poirier, 2012; Jorro, Maire Sandoz et Watrelot, 2012; Morrissette, Mottier Lopez et Tessaro, 2012).

La formation a également été conçue dans une perspective de «durabilité» afin que ses bénéfices ne se limitent pas aux participants directs, mais qu'ils soient également accessibles à leurs collègues actuels et futurs. Les enseignants ont donc été invités à mobiliser les savoirs de formation nouvellement construits dans la production d'activités d'apprentissage originales rendues disponibles en ligne. Les participants à ce projet de formation continue se sont donc formés à l'évaluation des apprentissages dans le but de pouvoir à leur tour former des pairs (enseignants). Dans cette perspective, le projet a été structuré en deux temps: un temps d'apprentissage pour soi (An 1) et un temps de conception d'activités d'apprentissage pour autrui (An 2). Cette double dimension constitue la finalité du projet de formation continue. Les 27 rencontres de formation d'une journée chacune ont été réparties de la façon suivante: trois rencontres communes aux deux sites portant sur l'analyse de besoins, dont l'une menée avec les conseillères et conseillers pédagogiques seulement; sur chacun des sites, une rencontre de définition des objectifs initiaux de formation, puis trois rencontres axées sur la construction de savoirs de référence; aussi, neuf rencontres (site A) et cinq rencontres (site B) pour la production d'activités d'apprentissage. Une rencontre de bilan a eu lieu sur chacun des sites. Enfin, la production de 13 activités d'apprentissage disponibles sur une plateforme interactive (Moodle) constitue le principal extrant de ce projet de formation continue.

\section{PROBLÉMATISATION THÉORIQUE}

Dans le cadre du projet de formation continue qui sert de contexte à notre étude, les enseignants, ainsi que Vial (2009) le mentionne à propos de toute personne non formée en évaluation, étaient soucieux d'acquérir des méthodes et des outils. Nous avons cependant fait le choix de travailler avec eux en amont de l'instrumentalisation et de ne pas orienter la formation sur l'appropriation d'outils ou de stratégies d'évaluation à appliquer. Dans cette perspective, la formation s'est appuyée sur un processus de recherche collaborative (Morrissette et al., 2012) qui «incite» chaque enseignant à endosser une posture de chercheur sur ses propres savoirs en évaluation. 
De manière générale, le développement professionnel des enseignants peut être défini comme:

[...] un processus graduel d'acquisition et de transformation des compétences et des composantes identitaires conduisant progressivement les individus et les collectivités à améliorer, enrichir et actualiser leur pratique, à agir avec efficacité et efficience dans les différents rôles et responsabilités professionnelles qui leur incombent, à atteindre un nouveau degré de compréhension de leur travail et à s'y sentir à l'aise (Portelance, Martineau et Mukamurera, 2014, p. 12).

Les indicateurs de ce développement sont une évolution dans les pratiques générées par un possible développement de compétences (Portelance, Martineau et Mukamurera, 2014, p. 12), l'accroissement d'une capacité réflexive et d'une intelligibilité de ses pratiques à ses propres yeux et aux yeux des pairs (Vial, 2009) ainsi que l'appropriation à différents degrés d'un agir professionnel de plus en plus porteur de sens et intentionnel (Vanhulle, 2013, 2014). Selon Vanhulle, l'appropriation de nouveaux savoirs professionnels contribue à un développement professionnel durable. Le développement professionnel de l'enseignant repose sur la construction de savoirs professionnels élaborés à partir de références multiples: son environnement et les pratiques qui l'entourent, sa formation initiale, les sources scientifiques, ses pairs et sa propre expérience. Un savoir professionnel résulte de l'agencement d'autres savoirs structurés dans un travail de conceptualisation et de régulation soutenu par une recherche de sens orientée vers des finalités propres à l'activité professionnelle du sujet. Les savoirs de référence désignent les savoirs définissant le métier; ils sont issus de sources autres que l'expérience de l'enseignant et ne se construisent pas nécessairement dans l'action, mais dans la réflexion. Les savoirs de référence proviennent de différentes sources: expérientielles, scientifiques, institutionnelles ou pratiques (Vanhulle, 2013). Ces savoirs agissent comme agents transformateurs de la réalité vécue en situation professionnelle, contribuant ainsi à l'élaboration de savoirs professionnels (Buysse, 2011). Dans ce processus, les savoirs de la formation, c'est-à-dire les savoirs abordés dans un contexte de formation continue, sont intériorisés et reconfigurés de manière constante au contact de nouvelles informations ou de nouvelles expériences et en fonction des contextes et des situations qui les sollicitent. À la suite de Vanhulle (2013), nous avons considéré que la construction de savoirs professionnels à partir de références théoriques est un enjeu essentiel pour le développement professionnel des enseignants, l'amélioration de la pratique évaluative s'appuyant sur l'intériorisation de ressources (concepts, stratégies de résolution de problèmes, de modes de raisonnement) et sur la réflexivité.

Selon Vial (2009), le développement de la compétence en évaluation implique de pouvoir problématiser des situations professionnelles. La problématisation de ces situations constitue un point de départ incontournable qui permet de cibler les contraintes situationnelles délimitant les besoins de formation des enseignants. Buysse (2011) estime pour sa part que le cadrage du problème et son recadrage à la 
lumière des savoirs de référence et d'expérience constituent un point d'ancrage pour la construction de savoirs professionnels. Dans le processus de formation choisi, nous avons donc délibérément encouragé une démarche de questionnement sur les connaissances que les enseignants mobilisent habituellement pour évaluer leurs élèves, sur l'identification des apprentissages professionnels qu'ils pensent devoir faire pour répondre aux nouvelles prescriptions ministérielles en matière d'évaluation (évaluer des compétences) et sur les manières de réaliser ces apprentissages, en tenant compte de leur volonté individuelle et collective de changement.

Dans le cadre d'une formation continue de type non applicatif, nous considérons que le concept de savoir professionnel favorise une meilleure compréhension des processus de réaménagement des ressources cognitives de l'enseignant. Le concept de savoir professionnel introduit également la possibilité de repérer comment les savoirs scientifiques ou institutionnels et les savoirs expérientiels se concurrencent ou s'appuient mutuellement dans une dynamique de coconstruction au sein de groupes de pairs ou lorsque les membres de communauté de pratique professionnelle et de communautés scientifiques travaillent ensemble (Morrissette, Mottier Lopez et Tessaro, 2012). La démarche réflexive mise en place dans les échanges qui ont ponctué la formation a représenté une occasion privilégiée d'observer les mécanismes par lesquels les participants développent une dynamique d'arbitrage et de négociation dans la construction de leurs savoirs professionnels.

La reconfiguration des savoirs de formation s'effectue dans le cadre d'interactions entre les enseignants, le chercheur-formateur et les conseillers pédagogiques. Il est donc possible de repérer dans les échanges certaines opérations «réflexivo-cognitives» des participants qui aident à comprendre quel sens ces derniers donnent aux savoirs abordés et quelle valeur ils leur attribuent (Vanhulle, 2013). Par exemple, on peut repérer des marques de jugement positif ou négatif, des justifications, des choix, des explications ou des attributions causales. Les activités réflexives engagent le soi professionnel de l'enseignant à apprécier les objets d'apprentissage ou les situations vécues en formation «en termes de valuation ${ }^{3}$ de rapports entre des fins et des moyens» (Vanhulle, 2013. p. 47). Autrement dit, l'enseignant pose en quelque sorte un jugement sur un savoir candidat en lui attribuant de la valeur en termes de rapports entre des fins souhaitées et des moyens de les atteindre (Vanhulle, 2013, citant Dewey, 2011). Le concept de valuation nous semble porteur pour comprendre les dynamiques collaboratives, car la question de l'interprétation des situations, des problématiques et des solutions y prend une place centrale et détermine largement l'acceptabilité des savoirs en circulation, quelle que soit leur origine, scientifique, situationnelle, prescriptive, expérientielle ou pratique. Ces éclairages théoriques

3. Le concept de valuation est utilisé par Vanhulle (2014) dans l'analyse de discours de professionnels en devenir. La valuation y est définie comme "un travail réflexif consistant à discuter et produire des valeurs » (p. 7). Ces valeurs se forment « dans des intentions volontaires, délibérées, critiques [...] consistant à "valuer" ce qui dans une situation existante crée une aversion; et ce qui, dans une situation possible à venir, crée une attirance» (Vanhulle, 2014, p. 7). 
nous ont poussée à émettre l'hypothèse que le repérage de la valeur attribuée aux savoirs de formation en contexte collaboratif permettrait de cerner des conditions d'acceptabilité de ces savoirs et donc leur potentiel effet dans le développement des compétences professionnelles. Nous interrogeant sur les qualités épistémologiques des savoirs et des connaissances construits dans le cadre du projet, nous avons établi des critères de validité et de viabilité des savoirs de référence sur lesquels s'appuyaient les participants pour accorder une valeur aux savoirs de formation. Ces critères ont été établis à la suite d'une recension d'écrits de didactique professionnelle, du champ de la professionnalisation, de l'épistémologie constructiviste et de la pédagogie (Nizet, 2016).

Ce cadre conceptuel nous permet de repérer des traces de valuation à travers des énoncés discursifs des participants. Nous avons catégorisé les valuations comme étant positives ou négatives en termes de justification de la valeur attribuée (validité) et de la conservation de cette valeur au fil des activités de formation (viabilité). Une valuation positive est associée à un savoir de formation lorsque le discours du participant présente des indices de son acceptation par la reprise d'information, l'acquiescement, le jugement positif, la reformulation, l'interprétation, l'inclusion dans un développement d'idées. Une valuation négative est associée à un savoir de formation lorsque le discours du participant présente des indices de refus, d'évitement, de jugement négatif, de doute ou de remise en cause du contenu d'une proposition antérieure d'un autre participant.

Afin de mieux comprendre les conditions de développement de la compétence à évaluer les apprentissages dans un contexte collaboratif, ce qui constitue l'objectif général de cette étude, nous souhaitons 1) décrire les problèmes de formation définis avec ces enseignants pour la compétence à évaluer durant le projet, 2) décrire les attributions de valeur données aux savoirs de formation au cours des deux années du projet pour les différents problèmes abordés afin de 3 ) présenter des pistes de réflexion quant aux conditions de développement de la compétence à évaluer.

\section{MÉTHODOLOGIE}

Le corpus analysé est constitué de matériel enregistré sur support audio ou vidéo provenant de deux rencontres ayant eu lieu pendant l'an 1 et d'une rencontre de l'an 2 sur le site A. L'analyse de contenu allait porter sur 67 segments, dont 38 segments sélectionnés dans un échange de $2 \mathrm{~h} 37$ min après un moment de formation plus formel durant la première année du projet, puis 29 segments provenant d'une séance de bilan de 55 minutes au courant de la deuxième année du projet.

Ce matériel a été traité selon une méthode d'analyse de contenu de discours. Nous avons d'abord procédé à un découpage des verbatims en séquences à partir d'un plan de travail reconstruit $a$ posteriori. Ce travail interprétatif de premier niveau a 
permis d'effectuer une sélection d'échanges représentatifs. Une fois cette scénarisation effectuée, il a été possible de repérer, dans l'ensemble des échanges retenus, des passages significatifs liés aux objets de l'étude en termes de problèmes de formation d'abord et de valuation des savoirs ensuite.

\section{RÉSULTATS}

Pour le premier objectif spécifique, nous décrirons les problèmes de formation soulevés par les enseignants au cours du projet afin de contextualiser les résultats d'analyse de valuations. Pour le deuxième objectif spécifique, nous présenterons les résultats de l'analyse des échanges pour les deux problèmes signalés, sous forme de données quantitatives pour les valuations repérées. Enfin, pour ce qui est du troisième objectif spécifique, nous illustrerons par des extraits de verbatims quelques cas de valuation à propos de certains savoirs de formation.

\section{Les besoins de formation}

Sur les deux sites, dès le début des rencontres de travail, nous avons constaté que les références scientifiques étaient presque totalement absentes du répertoire cognitif des enseignants pour désigner leurs gestes évaluatifs dans le nouveau contexte de l'approche par compétences. Il a été collectivement décidé de mettre l'accent sur la construction de ressources conceptuelles pour rehausser le niveau d'intelligibilité des échanges entre participants concernant la thématique de l'évaluation des compétences des élèves adultes. La réponse à ce besoin de formation a nécessité l'apprentissage d'éléments théoriques concernant le processus d'apprentissage de l'élève qui se sont révélés incontournables pour la poursuite de la formation. Il s'agit d'un processus dont les enseignants se sont révélés avoir une connaissance partielle d'un point de vue scientifique, mais fortement marquée par leur expérience professionnelle. La compréhension commune du processus d'apprentissage de l'élève a donc été reconnue comme un premier besoin de formation auquel répondre. $\mathrm{Au}$ terme de cette étape, sur le site $\mathrm{A}$, une équipe a décidé de concevoir du matériel de formation pour les pairs portant sur le processus d'apprentissage lui-même, sous forme d'une analogie. À la suite de ces apprentissages théoriques, une équipe du site $\mathrm{A}$ a déterminé un deuxième besoin de formation, soit celui de se former aux pratiques d'évaluation qui favorisent l'apprentissage. Dans la démarche de production de matériel d'apprentissage pour les pairs, cette équipe a produit des vidéos commentées où elle situe les interventions d'enseignants pilotant des démarches d'évaluation formative en se référant au processus d'apprentissage pour diagnostiquer les difficultés d'élèves avec qui ces enseignants interagissent en classe. 


\section{La valuation des savoirs de formation}

L'analyse de contenu des échanges de deux rencontres ayant eu lieu sur le site A a permis de confirmer ce que nous avions pu observer de manière empirique au cours des réunions de travail.

Comme le montre le tableau 1, les valuations attribuées aux savoirs de formation sur le processus d'apprentissage ont été en majorité positives (58\%), mais les valuations négatives demeurent importantes (42\%). L'analyse des valuations au cours de l'an 2 montre en effet que les poids des valeurs positives et négatives s'inversent.

\section{Tableau 1. Poids des valuations positives et négatives attribuées aux savoirs de} formation (An 1 et an 2, site A)

\begin{tabular}{|l|c|c|}
\hline Nature des valuations & An 1 (\%) & An 2 (\%) \\
\hline Positives & 58 & 29 \\
\hline Négatives & 42 & 71 \\
\hline Total & 100 & 100 \\
\hline
\end{tabular}

Lorsqu'on considère comment se concrétisent ces valuations en fonction des quatre critères retenus dans le cadre conceptuel (voir le tableau 2), on remarque que les valuations positives visent essentiellement le critère d'intelligibilité, ce qui n'est pas étonnant puisque le besoin de formation résultait essentiellement d'une perception d'inintelligibilité collective. Il est cependant intéressant de noter que les valuations négatives visent d'abord la crédibilité des savoirs de formation, et ensuite la légitimité et l'efficacité. Nous illustrerons ces constats par des extraits de verbatims dans la section suivante.

Tableau 2. Répartition des valuations positives et négatives en fonction de critères de validité et de viabilité (An 1 et an 2, site A)

\begin{tabular}{|l|c|c|c|c|}
\hline Valuations & Positives & Négatives & Positives & Négatives \\
\hline Crédibilité & 2,3 & 16,5 & 3,5 & 11 \\
\hline Intelligibilité & 44,5 & 10,5 & 18,5 & 15 \\
\hline Efficacité & 11 & 13 & 7 & 34 \\
\hline Légitimité & 0 & 2,5 & 0 & 11 \\
\hline Total & 58 & 43 & 29 & 71 \\
\hline \multicolumn{2}{|c|}{100} & \multicolumn{2}{|c|}{100} \\
\hline
\end{tabular}


Si le critère d'efficacité semble être une source de valuation négative durant la première année du projet, cela nous apparaît compréhensible du fait que le groupe était impliqué dans un travail intense de construction de savoirs de référence de source théorique, pour soi.

\section{La crédibilité des savoirs à construire: le poids des prescriptions et de l'expérience}

Après avoir défini le besoin de formation sur le processus d'apprentissage à la suite de l'élaboration d'une carte conceptuelle à partir de deux lectures à caractère scientifique au début de la première année du projet, nous avons fait un exposé sur quelques connaissances relatives au processus d'apprentissage. Nous avons employé à cette fin des termes utilisés dans un document ministériel encadrant l'évaluation des apprentissages: l'acquisition, la compréhension, l'application et la mobilisation de connaissances. Cet ancrage dans un document dont le contenu est prescriptif a justifié la démarche théorique, considérée a priori comme peu utile par les enseignants. L'objectif était d'aider les enseignants à discriminer ces quatre concepts considérés par eux comme étant presque équivalents, mais aussi et surtout à voir leur utilité dans un raisonnement pédagogique en évaluation des apprentissages. Par exemple, la distinction entre l' «application de connaissances» et la «mobilisation de connaissances " permet de discerner une approche par objectifs d'une approche par compétences dans laquelle la mobilisation des connaissances est un enjeu d'apprentissage crucial pour les élèves.

Les enseignants ont été invités à réaliser une activité portant sur les distinctions entre ces quatre concepts et sur les liens que ceux-ci entretiennent dans le processus d'apprentissage. Une démarche d'étayage a ensuite eu lieu au cours d'un échange informel dans lequel les enseignants, les conseillers pédagogiques et la chercheuseformatrice ont joué un rôle de régulation.

L'un des éléments clés de ces échanges est constitué d'interventions d'enseignants qui se fondent sur leur expérience pour nommer les distinctions entre les concepts d'application et de transfert. Le concept de transfert a été utilisé spontanément par des enseignants pour désigner la difficulté des élèves à dépasser le niveau de l'application décontextualisée de connaissances souvent privilégié dans leurs cahiers d'apprentissage; le concept de transfert semblait en effet bien connu des enseignants. C'est donc à travers des savoirs d'expérience que les enseignants ont en quelque sorte appuyé la crédibilité des concepts d'application et de transfert/mobilisation. 


\section{Des savoirs de référence en quête de légitimité}

La construction de ces savoirs de référence a demandé plusieurs mois, si l'on considère le temps écoulé du début de l'appropriation des concepts jusqu'à leur intégration dans des activités d'apprentissage pour autrui. À la suite de cette prise de conscience que l'application de connaissances ne représente plus la finalité ultime des apprentissages en FGA, les enseignants ont évoqué plusieurs facteurs qui pourraient, selon eux, freiner, voire empêcher la mise en œuvre cette nouvelle exigence du programme dans leur contexte professionnel. Cela nous a permis d'observer qu'un concept ne devient légitime pour les enseignants que s'il peut servir de référence dans l'exercice d'une pratique en situation.

Ainsi, une enseignante propose une généralisation tirée de son expérience pour indiquer qu'il ne lui semble pas possible que les élèves de FGA puissent tous être préparés à réussir le transfert ou la mobilisation des apprentissages, considérés comme trop complexes pour ceux-ci. Elle renvoie alors à son contexte professionnel, notamment aux difficultés et aux troubles d'apprentissages des élèves, pour justifier le fait que ce savoir de formation, tout en étant pertinent selon elle, ne résistera pas à son utilisation dans le contexte de sa classe de mathématiques. Seuls, d'après elle, «les élèves qui réussissent bien vont peut-être effectuer la deuxième partie du transfert, parce qu'ils vont pouvoir avoir un raisonnement...». Une autre enseignante fait le constat que le programme actuel lui permet d'éviter de travailler l'étape de mobilisation des connaissances avec les élèves, puisque, "présentement, on est encore par objectifs. Ce qu'on enseigne, c'est toujours acquisition, compréhension, application. Pis à la fin, là, on va à l'examen». Le matériel d'apprentissage dont dépend l'autoformation des élèves constitue selon une autre enseignante un obstacle à la mise en œuvre de la «mobilisation de connaissances» par les élèves, puisque «même les ouvrages de certaines maisons d'édition [...] restent à l'application encore [et] ne mènent pas beaucoup vers la tâche complexe [...] ». Enfin, une troisième enseignante tente d'expliquer ces limites par des facteurs psychologiques propres au vécu de certains élèves pour qui il ne serait «pas facile, voire impossible pour eux d'aller vers le transfert». Elle va jusqu'à invoquer des raisons culturelles pour justifier que «ce n'est comme tellement pas dans leur culture scolaire... On a beau essayer de leur montrer, ils vont refuser d'aller vers le transfert et ça reste dans l'application", ce qui empêcherait les élèves de transférer leurs apprentissages au moment des prétests préparatoires à l'examen final.

Une participante précise également que le changement est perçu comme une dépossession du rôle actuel de l'enseignant, qui serait en quelque sorte déchu de sa place «en avant» (de la classe) pour prendre une place «à côté» de l'élève (l'accompagner), ce qui pour elle équivaut à "changer notre rôle». Selon elle, les enseignants ont été «beaucoup freinés par ça». Cette intervention montre que l'incorporation de nouveaux savoirs de formation semble présenter pour certains enseignants un risque identitaire. Enfin, pour plusieurs enseignants, la compréhension des concepts liés au 
processus d'apprentissage paraît déconnectée de l'expérience ou de la pratique et est "encore abstrait» même s' 'il y en a pour qui c'est un peu plus clair».

Lorsque ces obstacles sont évoqués et discutés, l'espace d'échange s'ouvre alors pour certains enseignants sur des tentatives d'usage référentiel du savoir dans l'élaboration d'une activité d'apprentissage pour autrui, ce qui soulève d'autres obstacles.

Créer une activité de formation pour faire comprendre le savoir de référence à autrui s'est avéré une tâche particulièrement contraignante pour certains participants, la crédibilité des savoirs de formation acquis pour soi étant à nouveau mise en doute: "On va effectivement former d'autres personnes et c'est difficile de trouver une définition pour les autres [...] Est-ce qu'on dit la bonne chose, est-ce qu'on dit ce qu'on veut dire? Et moi, ça me dérange beaucoup.»

Cependant, le travail collaboratif rend aussi perceptibles les différences de degré d'appropriation des savoirs de formation, certains enseignants n'hésitant pas à anticiper l'usage de ces savoirs dans une nouvelle pratique, notamment ici en partageant l'analyse d'une situation vécue avec une élève à l'aide des savoirs de formation abordés:

Je pensais à une élève qui est en train de travailler sur la rédaction d'un reportage, mais il faut qu'elle passe par tout le processus, beaucoup d'acquisition, de compréhension et d'application; et éventuellement elle va arriver à la mobilisation. Et je me disais que ce que je dois faire c'est, pendant qu'elle fait ça, [identifier] elle est dans quoi.

Elle conclut en indiquant que ces concepts lui permettent de comprendre l'utilité de pratiques d'évaluation en aide à l'apprentissage afin de mieux préparer les élèves « $a u$ niveau de la mobilisation, parce qu'il ne peut pas juste en avoir dans le sommatif si on veut qu'ils apprennent à mobiliser». L'intelligibilité des savoirs de référence semble donc acquise pour cette enseignante qui peut en anticiper l'usage professionnel (critère d'efficacité).

\section{DISCUSSION}

La définition collaborative des problèmes de formation est un processus lent et complexe, dans lequel les participants ont progressivement appris à partager leurs perceptions des contraintes de leurs situations professionnelles et à en dégager une compréhension commune pour chercher des espaces d'autonomie au-delà d'un sentiment initial d'impuissance ou de résistance.

L'analyse a posteriori d'échanges qui ont eu lieu dans le cadre de cette formation continue a permis de repérer quelques conditions favorisant le développement des compétences des enseignants. Une compréhension fine des mécanismes qui soutiennent ou entravent la construction de savoirs professionnels permet d'observer 
que cette construction soulève des problèmes de valuation des savoirs de formation, notamment par un questionnement implicite sur la crédibilité et la légitimité de ces savoirs au sein du groupe des formés et plus largement au sein de leur communauté professionnelle (Bednarz, 2013).

Nous pouvons penser qu'une des premières conditions favorables au développement de compétences en évaluation consiste à reconnaître le degré de «littératie ${ }^{4}$ » des enseignants en matière d'évaluation au début de leur formation, c'est-à-dire l'état de leurs savoirs professionnels en évaluation, tels qu'ils sont définis par leur expérience, leur culture professionnelle et leur contexte de travail (Mertler, 2009). La nécessité de mettre l'accent sur le développement de ces connaissances de base en évaluation, de nature souvent intuitive et expérientielle, pour lui donner un caractère plus universel passe par l'apprentissage professionnel d'une terminologie commune afin de favoriser la communication entre pairs et l'intelligibilité des pratiques. Cela constitue une démarche de développement professionnel souvent considérée comme secondaire et négligeable par les personnes non formées en évaluation. Les apprentissages professionnels de ce type se révèlent extrêmement chronophages dans la formation continue, mais ils sont déterminants pour l'élaboration de savoirs pratiques intelligibles par autrui.

Une deuxième condition favorable consiste selon nous à interpréter les obstacles évoqués en cours d'appropriation comme des signes de questionnement épistémologique sur la valeur des savoirs coconstruits (Desgagné, 2007). La construction de savoirs professionnels dont les sources de référence sont parfois conflictuelles (Legendre et Morrissette, 2013) implique une démarche constante d'attribution de valeur selon des critères implicites de crédibilité, d'intelligibilité et de légitimité (Nizet et Leroux, 2015). La dimension épistémologique est donc constamment présente dans les délibérations des enseignants qui construisent ces savoirs en présence de pairs et de membres de communautés scientifiques.

Enfin, la décision de demander aux enseignants de construire du matériel de formation pour leurs pairs de manière presque concomitante à leur propre formation constitue une voie peu habituelle pour eux. Cette dimension du projet a permis de constater que la valeur des savoirs de référence considérés comme crédibles, intelligibles et légitimes par les participants qui se les sont appropriés est remise en doute lorsque vient le temps de les partager avec des pairs qui n'ont pas participé au processus de formation. En cela, le développement professionnel dépasse l'adhésion individuelle de l'enseignant à un projet de développement de compétences dans des situations pédagogiques isolées. Les obstacles mentionnés par les enseignants de FGA dans leur processus d'appropriation montrent que l'«amélioration» de la compétence à évaluer les apprentissages ne semble possible qu'à une troisième condi-

4. Traduction libre du terme "assessment literacy». 
tion favorable, celle de leur accompagnement dans la traversée de contraintes situationnelles sur lesquelles ils peuvent avoir prise.

\section{CONCLUSION}

Le pilotage d'un processus de formation non applicatif centré sur la réalité des enseignants, auquel se greffe un processus de recherche collaborative, permet de cerner la façon dont des savoirs produits par et pour la communauté professionnelle deviennent des ressources cognitives nouvelles, potentiellement mobilisables par les enseignants. Cela apparaît d'autant plus que le processus de formation rassemble, dans le cadre de partenariats universités-écoles, des membres de communautés scientifiques et de pratique pour qui les conditions de production des savoirs ne sont pas nécessairement semblables.

\section{Références bibliographiques}

BEDNARZ, N. (2013). Recherche collaborative et pratique enseignante. Regarder ensemble autrement. Paris: L'Harmattan.

BOUDJAOUI, M. et CLÉNET, J. (2011). Les enjeux d'une pédagogie de l'alternance dans la formation des enseignants. Les apports d'expériences françaises de formation et de formateurs d'adultes. Dans P. Maubant, J. Clénet et D. Poisson (dir.), Débats sur la professionnalisation des enseignants. Les apports de la formation des adultes (p. 13-56). Québec: Presses de l'Université du Québec.

BUYSSE, A. (2011). Une modélisation de la régulation et des médiations dans la construction des savoirs professionnels des enseignants. Dans P. Maubant et S. Martineau (dir.), Fondements des pratiques professionnelles des enseignants (p. 267-308). Québec: Presses de l'Université du Québec.

CONSEIL SUPÉRIEUR DE L'ÉDUCATION. (2014). Le développement professionnel, un enrichissement pour toute la profession enseignante. Avis au ministre de l'Éducation, du Loisir et du Sport et ministre de l'Enseignement supérieur, de la Recherche et de la Science. Québec: Gouvernement du Québec.

DESGAGNÉ, S. (2007). Le défi de coproduction de "savoir» en recherche collaborative: autour d'une démarche de reconstruction et d'analyse de récits de pratique enseignante. Dans M. Anadón (dir.), La recherche participative: multiples regards (p. 89-121). Québec: Presses de l'Université du Québec. 
DESGAGNÉ, S., BEDNARZ, N., LEBUIS, P., POIRIER, L. et COUTURE, C. (2001). L'approche collaborative de recherche en éducation: un rapport nouveau à établir entre recherche et formation. Revue des sciences de l'éducation, 27(1), 3364.

DUCHARME, P., LEBLANC, R., BOURASSA, M. et CHEVALIER, J. (2007). La recherche collaborative en milieu scolaire: un travail d'acculturation. Éducation et francophonie, 35(2), 217-232.

DURAND, M.-J. et POIRIER, S. (2012). La recherche collaborative au service du développement professionnel en ligne. Éducation et francophonie, 40(1), p. 119137.

FONTAINE, S., SAVOIE-ZAJC, L. et CADIEUX, A. (2013). L'impact des CAP sur le développement de la compétence des enseignants en évaluation des apprentissages. Éducation et francophonie, 41(2), 10-34.

GOUVERNEMENT DU QUÉBEC. (1999). Orientations pour la formation continue du personnel enseignant. Choisir plutôt que subir. Québec: Ministère de l'Éducation.

GOUVERNEMENT DU QUÉBEC. (2003). Politique d'évaluation des apprentissages. Québec: Ministère de l'Éducation.

GOUVERNEMENT DU QUÉBEC. (2015). Programme de la formation générale des adultes. Québec: Ministère de l'Éducation, de l'Enseignement supérieur et de la Recherche. Récupéré de http://www.education.gouv.qc.ca/adultes/suivre-un-programmedetudes/formation-generale-des-adultes/

JORRO, A., MAIRE SANDOZ, M.-O. et WATRELOT, M. (2012). Recherche collaborative et développement professionnel des acteurs. Dans Le travail enseignant au XXI siècle. Perspectives croisées: didactiques et didactique professionnelle. Actes du colloque international de didactique HGEC, 16, 17 et 18 mars 2011, INRP, Lyon. Récupéré de http://www.inrp.fr/archives/colloques/travail-enseignant/contrib/ 27.pdf

LEGENDRE, M.-F. et MORRISSETTE, J. (2013). Défis et enjeux de l'approche par compétences dans un contexte d'obligation de résultats. Dans J. Morrissette et M.-F. Legendre (dir.), Enseigner et évaluer. Regards sur les enjeux éthiques et sociopolitiques (p. 211-246). Québec: Presses de l'Université Laval.

MERTLER, C. A. (2009). Teachers' assessment knowledge and their perceptions of the impact of classroom assessment professional development. Improving Schools, 12(2), 101-113. doi: 10.1177/1365480209105575 
MORRISSETTE, J., MOTTIER LOPEZ, L. et TESSARO, W. (2012). La production de savoirs négociés dans deux recherches collaboratives sur l'évaluation formative. Dans L. Mottier Lopez et G. Figari (dir.), Modélisations de l'évaluation en éducation: questionnements épistémologiques (p. 27-43). Bruxelles, Belgique: De Boeck.

NIZET, I. (2014). Formation des enseignants du secteur de l'éducation des adultes à l'évaluation des compétences: défis et perspectives. Revue des sciences de l'éducation, 40(2), 373-396.

NIZET, I. (2016). Transitions de culture évaluative chez des futurs enseignants de l'enseignement secondaire. Phronesis, 5(3-4).

NIZET, I. et LEROUX, J.-L. (2015). La construction de savoirs conceptuels en évaluation: enjeux pour un développement professionnel en contexte de formation continue. e-JIREF. Évaluer. Journal international de recherche en éducation et formation.

PORTELANCE, L., MARTINEAU, S. et MUKAMURERA, J. (2014). Développement et persévérance professionnels dans l'enseignement. Québec: Presses de l’Université du Québec.

VANHULLE, S. (2013). Dialoguer avec les discours des professionnels en devenir. Ikastaria, 19, 37-67.

VANHULLE, S. (2014). Comprendre le développement professionnel par l'analyse des discours. Récupéré de www.forumlecture.ch/myUploadData/files/2014_1_Vanhulle.pdf

VIAL, M. (2009). Se former pour évaluer. Se donner une problématique et élaborer des concepts ( $2^{\mathrm{e}}$ éd.). Bruxelles, Belgique: De Boeck.

VINATIER, I. (2015). Recherche collaborative. Dans A. Jorro (dir.), Dictionnaire des concepts de la professionnalisation (p. 249-252). Louvain-la-Neuve, Belgique: De Boeck. 